\title{
MOTION CONTROL OF A TENSEGRITY PLATFORM*
}

\author{
NARONGSAK KANCHANASARATOOL ${ }^{\dagger}$ AND DARRELL WILLIAMSON ${ }^{\ddagger}$
}

\begin{abstract}
In this paper, we develop a passive nonlinear constrained particle dynamic model for a class of tensegrity platform structures. Based on the steady state input-output mapping, we then formulate a neural network inversion problem which is later used as the basis for the design of large scale path tracking algorithms.
\end{abstract}

1. Introduction. Tensegrity structures (so named by Kenneth Snelson in 1948 because of their integrity under tension) can be thought of as structures in which the shape is guaranteed by the interaction between a continuous network of members in tension and a set of isolated members in compression. Investigation of the mathematical properties remain an interest and a challenge [1], [2], and provide exciting opportunities for new civil and mechanical applications [3]. We shall refer to tension members as strings, compression members as bars, and the ends of bars as nodes. In [4], a class I tensegrity structure is defined as one in which only one bar is connected to any node, while a class II structure is one in which at least one node has more than one bar connected to it. However, the distinguishing feature of all tensegrity structures is that a continuous connection between any two nodes by traversing only along the bars is not possible.

In [5], [6], the topology of a particular type of class I tensegrity structures having $N$ bars, $S$ strings and $M$ stages with $P_{k}$ strings in stage $k$ was described. Then in [7], a description of these structures in terms of a constrained mass-spring particle dynamic model was considered. In particular, a constrained particle dynamic model was developed in which each bar was represented by two particles positioned at the ends of the bar separated by a distance equal to the length of the bar. A method for regulating the position and orientation of the resulting constrained mass-spring model with respect to a given equilibrium point was presented based on linearization of the resulting nonlinear differential equations.

In this paper, we begin by defining the general structure of a tensegrity platform as a particular class II tensegrity structure in which at least half the nodes have only one bar attached. In section 2, a passive nonlinear constrained particle dynamic (or mass-spring) model is developed as the basis for designing a system for controlling the position and orientation of the structure along a prescribed path by adjusting the lengths of the bars. Then in section 3, we formulate a neural network "inversion

\footnotetext{
*Received on March 14, 2002; accepted for publication on September 10, 2002.

$\dagger$ Australian National University, Faculty of Engineering \& Information Technology, Canberra, ACT, 0200 AUSTRALIA. E-mail: Narongsak.Kanchanasaratool@faceng.anu.edu.au

${ }_{\ddagger}^{\ddagger}$ University of Wollongong, Faculty of Informatics, Wollongong, NSW 2522 AUSTRALIA. E-mail: Darrell_Williamson@uow.edu.au
} 
problem" which seeks the (approximate) constant input signal $\overline{\mathbf{u}}$ to give a desired steady state output response $\overline{\mathbf{y}}$. The performance of two path tracking algorithms; a quasi-static algorithm based on an open loop piecewise constant input, and a gain scheduling algorithm based on an interpolation of "locally designed" controllers, are then examined. An early version of these results has appeared [8], [9].

2. Tensegrity Platform. A $\left(N, S ; P_{1}, P_{2}, \ldots, P_{M}\right)$ class I structure described in [5],[6] has a total of $N$ bars, $S$ strings and $P_{k}$ bars in stage $k$ (with $\sum P_{k}=N$ ). The structure is said to symmetrical if $P_{k}=P$ for all $k$; otherwise, the structure is said to be non-symmetrical.

Without any loss of generality, we henceforth assume that the $N$ bar connections occur between nodes $\mathbf{p}_{2 k-1}$ and $\mathbf{p}_{2 k}$ for $k=1,2, . ., N$. We indicate when a string connection exists between node $\mathbf{p}_{i}$ and node $\mathbf{p}_{j}$ by writing $[i, j]$. The following results are of interest. The cited references provide general expressions for determining all the spring connections.

Theorem 2.1. [5],[6]

(a) The single stage $\left(N, S ; P_{1}\right)$ tensegrity has $N=P_{1}$ bars and $S=3 N$ strings. The nodes which define both the base and top form a $N$-sided polygon. Each node is connected by 3 springs to 3 other nodes.

(b) A symmetrical $(N, S ; P, P, \ldots P)$ tensegrity structure with $M>1$ stages has $N=M P$ bars and $S$ strings where $S=4 N-2($ for $P=2)$ and $S=4 N($ for $P>2)$. Each node is connected by 4 springs to 4 other nodes.

(c) An $\left(N, S ; P_{1}, P_{2}, \ldots P_{M}\right)$ tensegrity structure exists when the following conditions are satisfied:

$$
\text { (i) } \quad P_{1}>1 \text { and } P_{k} \geq 1 \text { for } k \geq 2 \quad \text { (ii) } \quad M=r \text { when } P_{r}=1
$$

$$
\text { (iii) } \quad\left|P_{k+1}-P_{k}\right|=1 \text { for } 1 \leq k \leq M-1
$$

The number $S$ of strings for all $\left(N, S ; P_{1}, P_{2} \ldots, P_{M}\right)$ structures is bounded by $3 N \leq S \leq 4 N$. In particular, for (a) symmetrical structures

$$
S=\left\{\begin{array}{ccc}
3 N & ; \quad M=1, P \geq 3 \\
4 N-2 & ; \quad M \geq 2, P=2 \\
4 N & ; \quad M \geq 2, P \geq 3
\end{array}\right.
$$

and for (b) non-symmetrical structures (and so $M \geq 2$ )

$$
S=4 N-L ; L=\sum_{k=1}^{M} \ell_{k}
$$

where 


$$
\begin{aligned}
& \ell_{1}=1 \text { if } P_{1}=2 \text { or } P_{2}=P_{1}-1 \\
& \ell_{k}=1 \text { for } 2 \leq k \leq M-1 \text { if } P_{k}=P_{k-1}+1 \text { and } P_{k+1}=P_{k-1} \\
& \ell_{M}= \begin{cases}1 & \text { if } P_{M}=2 \text { or } P_{M}=P_{M-1}+1 \\
2 & \text { if } P_{M}=1\end{cases}
\end{aligned}
$$

Each node is connected by either 3 or 4 springs to either 3 or 4 other nodes.

We make the following definitions.

Definition 2.1. (i) The base nodes of an $\left(N, S ; P_{1}, P_{2} \ldots, P_{M}\right)$ tensegrity structure are defined as the $P_{1}$ nodes $\left\{\mathbf{p}_{b_{1}}, \mathbf{p}_{b_{3}}, \ldots, \mathbf{p}_{b_{2 P_{1}-1}}\right\}$ which are a subset of the $2 P_{1}$ nodes in stage 1 that are connected to each other only by springs called the base strings. The base bars are the $P_{1}$ bars defined by the node pairs $\left\{\mathbf{p}_{b_{2 k-1}}, \mathbf{p}_{b_{2 k}}\right\}$ for $k=1,2, . ., P_{1}$.

(ii) The top nodes of a $\left(N, S ; P_{1}, P_{2} \ldots, P_{M}\right)$ tensegrity structure are defined as the $P_{M}$ nodes $\left\{\mathbf{p}_{t_{1}}, \mathbf{p}_{t_{3}}, \ldots, \mathbf{p}_{t_{2 P_{M}-1}}\right\}$ which are a subset of the $2 P_{M}$ nodes in stage $M$ that are connected to each other only by springs called the top strings. The top bars are the $P_{M}$ bars defined by the node pairs $\left\{\mathbf{p}_{t_{2 k-1}}, \mathbf{p}_{t_{2 k}}\right\}$ for $k=1,2, . ., P_{M}$.

(iii) Define a platform to be any (finite area) 2-dimensional surface. Then for $P_{1} \geq 3$ and $P_{M} \geq 3$, an $\left(N, S ; P_{1}, P_{2}, \ldots P_{M}\right)$ tensegrity platform is defined from a class $I\left(N, S ; P_{1}, P_{2}, \ldots P_{M}\right)$ tensegrity structure by removing the base and top strings, and fixing the base bars to a base platform at the base nodes, and the top bars to a top platform at the top nodes using spherical joints.

For simplicity, we henceforth refer to the base platform as the base, and the top platform as the top of the tensegrity platform structure.

2-stage 6-bar Platform: A 2-stage 6-bar tensegrity platform can be obtained from the 2 -stage $(6,24 ; 3,3)$ tensegrity structure shown in Fig 1 by replacing the top nodes $\left\{p_{1}, p_{3}, p_{5}\right\}$ and the springs connecting top nodes $\left\{p_{1}, p_{3}\right\},\left\{p_{1}, p_{5}\right\}$ and $\left\{p_{3}, p_{5}\right\}$ by a top (platform), and fixing the base nodes $\left\{p_{7}, p_{9}, p_{11}\right\}$ to the base (platform). The three top bars are attached to the top at the top nodes $\left\{\mathbf{p}_{1}, \mathbf{p}_{3}, \mathbf{p}_{5}\right\}$ and three base bars are attached to the base at the base nodes $\left\{\mathbf{p}_{7}, \mathbf{p}_{9}, \mathbf{p}_{11}\right\}$ using spherical joints. The resulting 2 -stage tensegrity platform with the 18 string connections: $\{[1,6],[1,8],[2,3],[2,8],[2,11],[2,12],[3,12],[4,5],[4,9],[4,10],[4,12],[5,10]$, $[6,7],[6,8],[6,10],[7,10],[8,11],[9,12]\}$ is illustrated in Fig 2.

Lemma 2.1. An $\left(N, S ; P_{1}, P_{2}, \ldots P_{M}\right)$ tensegrity platform is a class II tensegrity structure in which all the base nodes and top nodes are geometrically constrained. For $M>1$, all $P_{1}\left(P_{M}\right)$ nodes in stage 1 (stage $M$ ) that are not base (top) nodes satisfy only 1 geometric constraint. For $3 \leq k \leq M-1$, all the $2 P_{k}$ nodes in stages 2 to $M-1$ satisfy only one geometric constraint.

The natural (or maximal) coordinates for a tensegrity platform consist of the 
Cartesian coordinates of each node (which includes the spherical joints). The generalized coordinates for this platform is a reduced set of independent coordinates which provides a mimimal parametrization of the structure from which the dynamic behavior of the structure can be determined. In order to derive a dynamic model for a tensegrity platform, we also use the fact that since all the base nodes and top nodes are spherical joints, no further kinematic constraints are introduced.

2.1. Number of Degrees of Freedom. Overall, the tensegrity platform is represented by $2 N$ particles $\left\{\mathbf{p}_{j} ; 1 \leq j \leq 2 N\right\}$ in which every particle defines a node. Each rigid bar is defined by two nodes $\left\{\mathbf{p}_{2 k-1}, \mathbf{p}_{2 k-1}\right\}$ and the base and top are described by $P_{1}$ and $P_{M}$ nodes respectively. Any platform has 6 DOFs, and so if $P$ nodes are positioned on a platform with no 3 collinear, then $3 P-6$ constraints are required. We have the following result

Lemma 2.2. Given two nodes $\left\{\mathbf{p}_{k}(t), \mathbf{p}_{m}(t)\right\}$ at time $t$, define the function

$$
h_{k, m}\left(\mathbf{p}_{k}(t), \mathbf{p}_{m}(t), t\right) \triangleq\left\|\mathbf{p}_{k}-\mathbf{p}_{m}\right\|^{2}-D_{k, m}(t) ; D_{k, m}(t) \triangleq L_{k, m}^{2}(t)
$$

where $\|\cdot\|$ denotes the Euclidean norm. For simplicity, we henceforth use the shorthand notation: $h_{k, m} \triangleq h_{k, m}\left(\mathbf{p}_{k}(t), \mathbf{p}_{m}(t), t\right)$ so that the constraints as defined by the bar lengths are described by $\left\{h_{2 k-1,2 k}=0 ; k=1,2, \ldots, N\right\}$.

(i) Assume that no three points on the base are collinear. Then a set of $3 P_{1}-6$ independent base node constraints are given by:

$$
\begin{aligned}
h_{b_{2 k-1}, b_{2 k+1}} & =0 ; \quad k=1,2, . ., P_{1}-1 \\
h_{b_{2 P_{1}-1}, b_{1}} & =0 \\
h_{b_{1}, b_{2 k+3}} & =0 ; \quad k=1,2, . ., P_{1}-3 \\
h_{b_{3}, b_{2 k+5}} & =0 ; \quad k=1,2, . ., P_{1}-3 .
\end{aligned}
$$

(ii) Assume that no three points on the top are collinear. Then a set of $3 P_{M}-6$ independent top node constraints are given by:

$$
\begin{aligned}
h_{t_{2 k-1}, t_{2 k+1}} & =0 ; \quad k=1,2, . ., P_{1}-1 \\
h_{t_{2 P_{1}-1}, t_{1}} & =0 \\
h_{t_{1}, t_{2 k+3}} & =0 ; \quad k=1,2, . ., P_{1}-3 \\
h_{t_{3}, t_{2 k+5}} & =0 ; \quad k=1,2, . ., P_{1}-3 .
\end{aligned}
$$

Since each particle is represented by 3 coordinates, the number $D$ of degrees-offreedom is given by $D=2 N \times 3-C=6 N-C$ where $C$ is the number of constraints on the nodes described as follows:

(i) There are $P_{M}$ nodes (with no 3 collinear) constrained to lie on the top platform which require $3 P_{M}-6$ constraints given by (2). Similarly, there are $P_{1}$ nodes constrained to lie on the base platform which require $3 P_{1}-6$ constraints given by (3). 
(ii) There are $N$ constraints representing the lengths of the bars given by

$$
h_{2 k-1,2 k}=0 ; k=1,2, . ., N \text {. }
$$

(iii) Finally, in a body frame which is attached to the base, the positions of the $P_{1}$ base nodes are fixed which gives a further $3+2+1=6$ constraints (corresponding to the number of zeros in (5) below). Without any loss of generality, we assume the base is the $x-y$ plane, and

$$
\mathbf{p}_{b_{1}}^{T}=[0,0,0] ; \mathbf{p}_{b_{2}}^{T}=\left[L_{b_{1}, b_{2}}, 0,0\right] ; \mathbf{p}_{b_{k}}^{T}=\left[\alpha_{x, k}, \alpha_{y, k}, 0\right] \text { for } k=3,4, . ., P_{1} .
$$

We summarize these facts as follows:

Lemma 2.3. In the tensegrity body frame, the $\left(N, S ; P_{1}, P_{2}, \ldots P_{M}\right)$ tensegrity platform has $D$ degrees-of-freedom where

$$
D=5 N+6-3 P_{1}-3 P_{M}
$$

Consequently, a minimal set of $2 D$ generalized coordinates describe its dynamic behavior.

For a 2-stage 6-bar platform where $N=6, M=2 ; P_{1}=P_{2}=3$, we have $D=18$.

2.2. Constrained Particle Dynamic Model. For a tensegrity platform, it is neither obvious nor convenient to develop a model in terms of the minimal generalized coordinates. Instead, we now develop a model to describe the motion of a tensegrity platform in terms of particles (ie point masses) that represent the ends of the bars which are subject to geometric constraints. As shown in [7], the dynamical equations for a tensegrity structure in which each bar is represented by two constrained particles subject to translational and Euler dynamics is equivalent (subject only to a change in the length and/or mass distribution) to an idealized (ie zero diameter) bar. A similar conclusion can be reached with respect to representing an idealized (ie zero thickness) platform in terms of constrained particles located on its surface. In practice, however, bars have non-zero diameter and platforms have non-zero thickness, and so the dynamical behaviour of the constrained particle dynamics model and a model derived by including inertial effects of the bars and plate will differ. Numerical results [19] however demonstrate that a particle dynamic formulation provides a very useful approximation of the motion of a tensegrity platform. It should also be noted that the steady state (or static) characteristics of the particle dynamic models provide an exact representation of position and orientation of tensegrity platforms, and tensegrity structures more generally.

Suppose $\mathbf{f}_{k}$ is the Newtonian force at node $k$ which occurs as a result of spring forces applied at point $k$. Then assuming a linear cable model, we have

$$
\mathbf{f}_{k}=\sum_{m}\left\{k_{m k}\left(\ell_{m k}-\ell_{m k}^{0}\right)+d_{m k} \dot{\ell}\right\} \mathbf{e}_{m k}
$$


in which $\left\{k_{m k}, d_{m k}\right\}$ are respectively the spring constants and the damping constants of the springs. The summation in (6) is over all nodes $m$ which are connected to node $k$ by a spring where $\ell_{m k}^{0}$ is the rest length of the spring, $\ell_{m k}$ is the length at time $t$ of this spring, and $\mathbf{e}_{m k}$ is the unit vector from node $m$ to node $k$ as given by

$$
\ell_{m k}=\left\|\mathbf{p}_{m}-\mathbf{p}_{k}\right\| ; \mathbf{e}_{m k}=\frac{\mathbf{p}_{m}-\mathbf{p}_{k}}{\ell_{m k}} .
$$

The motion of each particle is subject to constraints (2) - (4), and in order to guarantee that the model constraints are satisfied, we follow an established approach in multibody systems analysis and add a constraint force $\hat{\mathbf{f}}_{j}$ to each node. The motion of the tensegrity platform in which each particle of unit mass is subject to both a spring force $\mathbf{f}_{k}$ and a constraint force $\hat{\mathbf{f}}_{k}$ is then given by

$$
\ddot{\mathbf{p}}_{k}=\mathbf{f}_{k}+\hat{\mathbf{f}}_{k} ; 1 \leq k \leq 2 N .
$$

Using the principle of virtual work, we now derive the explicit form of the constraint forces.

2.2.1. Principle of Virtual Work. Since constraint forces influence accelerations, these forces must convert the accelerations of the particles into "legal" accelerations that are consistent with the constraints. Physical considerations also impose two further conditions: (i) the constraint forces must obey Newton's third law, and (ii) for stationary constraints (i.e when $D_{k m}(t)$ in (1) is constant for all $t$ ), the constraint forces must neither add nor remove energy from the system. This latter requirement is referred to as the principle of virtual work [12],[13]. Constraint forces which satisfy this property also provide a model for the internal forces exerted in the bars. We have the following result.

TheOREM 2.2. The constraint forces $\hat{\mathbf{f}}_{k}$ which act on the unit masses at nodes $\mathbf{p}_{k}$ and satisfy the principle of virtual work are of the form

$$
\hat{\mathbf{f}}_{k}=\sum_{m} \lambda_{k, m}\left(\mathbf{p}_{k}-\mathbf{p}_{m}\right) ; \lambda_{k, m}=\lambda_{m, k}
$$

for some Lagrange parameters $\left\{\lambda_{k, m}\right\}$ where the summation is over all nodes $m$ for which there is a geometric constraint $h_{k, m}=0$ between node $m$ and node $k$. In particular:

(i) Suppose $M \geq 3$, and the two nodes $\left\{\mathbf{p}_{2 i-1}, \mathbf{p}_{2 i}\right\}$ represent a bar in stage $k$ for $2 \leq k \leq M-1$. Then the constraint forces $\left\{\hat{\mathbf{f}}_{2 i-1}, \hat{\mathbf{f}}_{2 i}\right\}$ are given in terms of the single Lagrange parameter $\lambda_{2 i-1,2 i}$ by

$$
\hat{\mathbf{f}}_{2 i-1}=\lambda_{2 i-1,2 i}\left(\mathbf{p}_{2 i-1}-\mathbf{p}_{2 i}\right) ; \hat{\mathbf{f}}_{2 i}=-\lambda_{2 i-1,2 i}\left(\mathbf{p}_{2 i-1}-\mathbf{p}_{2 i}\right) .
$$

(ii) The constraint forces $\left\{\hat{\mathbf{f}}_{b_{j}} ; j=1,2, . ., 2 P_{1}\right\}$ on the $P_{1}$ base nodes $\left\{\mathbf{p}_{b_{2 k-1}} ; k=\right.$ $\left.1,2, . ., P_{1}\right\}$ and the $P_{1}$ base bar nodes $\left\{\mathbf{p}_{b_{2 k}} ; k=1,2, . ., P_{1}\right\}$ corresponding to the base 
node constraints (2) are given in terms of $4 P_{1}-6$ Lagrange parameter by

$$
\begin{aligned}
\hat{\mathbf{f}}_{b_{2 k}} & =-\lambda_{b_{2 k-1}, b_{2 k}}\left(\mathbf{p}_{b_{2 k-1}}-\mathbf{p}_{b_{2 k}}\right) ; k=1,2, \ldots, P_{1} \\
\hat{\mathbf{f}}_{b_{1}} & =\sum_{k=1}^{P_{1}-1} \lambda_{b_{1}, b_{2 k+1}}\left(\mathbf{p}_{b_{1}}-\mathbf{p}_{b_{2 k+1}}\right)+\lambda_{b_{1}, b_{2}}\left(\mathbf{p}_{b_{1}}-\mathbf{p}_{b_{2}}\right) \\
\hat{\mathbf{f}}_{b_{3}} & =\sum_{k=1}^{P_{1}-2} \lambda_{b_{3}, b_{2 k+3}}\left(\mathbf{p}_{b_{3}}-\mathbf{p}_{b_{2 k+3}}\right)+\lambda_{b_{3}, b_{1}}\left(\mathbf{p}_{b_{3}}-\mathbf{p}_{b_{1}}\right)+\lambda_{b_{3}, b_{4}}\left(\mathbf{p}_{b_{3}}-\mathbf{p}_{b_{4}}\right)
\end{aligned}
$$

and: (a) For $P_{1}=3$

$$
\hat{\mathbf{f}}_{b_{5}}=\sum_{k=1}^{2} \lambda_{b_{5}, b_{2 k-1}}\left(\mathbf{p}_{b_{5}}-\mathbf{p}_{b_{2 k-1}}\right)+\lambda_{b_{5}, b_{6}}\left(\mathbf{p}_{b_{5}}-\mathbf{p}_{b_{6}}\right) ;
$$

(b) For $P_{1} \geq 4$

$$
\begin{aligned}
\hat{\mathbf{f}}_{b_{5}}= & \sum_{k=1}^{2} \lambda_{b_{5}, b_{2 k-1}}\left(\mathbf{p}_{b_{5}}-\mathbf{p}_{b_{2 k-1}}\right)+\lambda_{b_{5}, b_{7}}\left(\mathbf{p}_{b_{5}}-\mathbf{p}_{b_{7}}\right)+\lambda_{b_{5}, b_{6}}\left(\mathbf{p}_{b_{5}}-\mathbf{p}_{b_{6}}\right) \\
\hat{\mathbf{f}}_{b_{2 P_{1}-1}}= & \sum_{k=1}^{2} \lambda_{b_{2 P_{1}-1}, b_{2 k-1}}\left(\mathbf{p}_{b_{2 P_{1}-1}}-\mathbf{p}_{b_{2 k-1}}\right)+\lambda_{b_{2 P_{1}-1}, b_{2 P_{1}-3}}\left(\mathbf{p}_{b_{2 P_{1}-1}}-\mathbf{p}_{b_{2 P_{1}-3}}\right) \\
& \quad+\lambda_{b_{2 P_{1}-1}, b_{2 P_{1}}}\left(\mathbf{p}_{b_{2 P_{1}-1}}-\mathbf{p}_{b_{2 P_{1}}}\right)
\end{aligned}
$$

(c) For $P_{1} \geq 5$, then for $m=1,2, . ., P_{1}-4$

$$
\begin{aligned}
& \hat{\mathbf{f}}_{b_{5+2 m}}=\sum_{k=1}^{2} \lambda_{b_{2 m+5}, b_{2 k-1}}\left(\mathbf{p}_{b_{2 m+5}}-\mathbf{p}_{b_{2 k-1}}\right)+\sum_{k=1}^{2} \lambda_{b_{2 m+5}, b_{2 m+4 k-1}}\left(\mathbf{p}_{b_{2 m+5}}-\mathbf{p}_{b_{2 m+4 k-1}}\right) \\
& +\lambda_{b_{2 m+5}, b_{6+2 m}}\left(\mathbf{p}_{b_{2 m+5}}-\mathbf{p}_{b_{2 m+6}}\right) .
\end{aligned}
$$

(iii) The constraint forces $\left\{\hat{\mathbf{f}}_{t_{j}} ; j=1,2, . ., 2 P_{M}\right\}$ on the $P_{M}$ top nodes $\left\{\mathbf{p}_{t_{2 k-1}} ; k=\right.$ $\left.1,2, . ., P_{M}\right\}$ and the $P_{M}$ top bar nodes $\left\{\mathbf{p}_{t_{2 k}} ; k=1,2, . ., P_{M}\right\}$ corresponding to the top node constraints (3) are given given in terms of $4 P_{M}-6$ Lagrange parameter similarly to (11)- (14) provide $P_{1}$ is replaced everywhere by $P_{M}$ and $b_{j}$ is replaced everywhere by $t_{j}$.

In order to prove this result, observe that the kinetic energy $T$ and rate of change $\dot{T}$ of the constrained mass-spring system as represented by the $2 N$ unit mass particles $\left\{\mathbf{p}_{k} ; 1 \leq k \leq 2 N\right\}$ are given by

$$
T=\frac{1}{2} \sum_{k=1}^{2 N}\left\|\dot{\mathbf{p}}_{k}\right\|^{2} ; \dot{T}=\sum_{k=1}^{2 N}\left(\ddot{\mathbf{p}}_{k}\right)^{T} \dot{\mathbf{p}}_{k} .
$$

Hence the rate of change $\dot{\hat{T}}$ in the kinetic energy due only to the constraint forces is given by

$$
\dot{\hat{T}}=\sum_{k=1}^{2 N} \hat{\mathbf{f}}_{k}^{T} \dot{\mathbf{p}}_{k} .
$$


Then using (9), it follows that

$$
\dot{\hat{T}}=\sum_{m} \sum_{k<m} \lambda_{k, m} \frac{d}{d t}\left(\left\|\mathbf{p}_{k}-\mathbf{p}_{m}\right\|^{2}\right)
$$

Now from (1)

$$
\dot{h}_{k, m}\left(\mathbf{p}_{k}, \mathbf{p}_{m}, t\right)=2\left(\mathbf{p}_{k}-\mathbf{p}_{m}\right)^{T}\left(\dot{\mathbf{p}}_{k}-\dot{\mathbf{p}}_{m}\right)-\dot{D}_{k, m}
$$

and so from (16), we have

$$
\dot{\hat{T}}=\sum_{m} \sum_{k<m} \lambda_{k, m}\left[\dot{h}_{k, m}+\dot{D}_{k, m}\right]
$$

For "legal" accelerations, $\dot{h}_{k, m}\left(\mathbf{p}_{k}, \mathbf{p}_{m}, t\right)=0$ and for constant constraints $\dot{D}_{k m}(t)=$ 0 , which then gives the result in (9). Part (ii) then follows from structural properties which define the tensegrity platform, while part (iii) follows from Lemma 2.2.

Corollary 2.1. The constraint forces $\left\{\hat{\mathbf{f}}_{m+j} ; 1 \leq m \leq 6,0 \leq j \leq 1\right\}$ of the 2-stage 6-bar tensegrity structure which act respectively on the unit masses at the tensegrity nodes and satisfy the principle of virtual work are given in terms of the 6 Lagrange parameters $\left\{\lambda_{2 i-1,2 i} ; 1 \leq i \leq 6\right\}$ for the top nodes by $j=0$ and the base nodes by $j=1$ where

$$
\begin{aligned}
& \hat{\mathbf{f}}_{1+j}=\lambda_{1+j, 2+j}\left(\mathbf{p}_{1+j}-\mathbf{p}_{2+j}\right)+\lambda_{1+j, 3+j}\left(\mathbf{p}_{1+j}-\mathbf{p}_{3+j}\right)+\lambda_{1+j, 5+j}\left(\mathbf{p}_{1+j}-\mathbf{p}_{5+j}\right) \\
& \hat{\mathbf{f}}_{2+j}=-\lambda_{1+j, 2+j}\left(\mathbf{p}_{1+j}-\mathbf{p}_{2+j}\right) \\
& \hat{\mathbf{f}}_{3+j}=\lambda_{3+j, 4+j}\left(\mathbf{p}_{3+j}-\mathbf{p}_{4+j}\right)-\lambda_{1+j, 3+j}\left(\mathbf{p}_{1+j}-\mathbf{p}_{3+j}\right)+\lambda_{3+j, 5+j}\left(\mathbf{p}_{3+j}-\mathbf{p}_{5+j}\right) \\
& \hat{\mathbf{f}}_{4+j}=-\lambda_{3+j, 4+j}\left(\mathbf{p}_{3+j}-\mathbf{p}_{4+j}\right) \\
& \hat{\mathbf{f}}_{5+j}=\lambda_{5+j, 6+j}\left(\mathbf{p}_{5+j}-\mathbf{p}_{6+j}\right)-\lambda_{1+j, 5+j}\left(\mathbf{p}_{1+j}-\mathbf{p}_{5+j}\right)-\lambda_{3+j, 5+j}\left(\mathbf{p}_{3+j}-\mathbf{p}_{5+j}\right) \\
& \hat{\mathbf{f}}_{6+j}=-\lambda_{5+j, 6+j}\left(\mathbf{p}_{5+j}-\mathbf{p}_{6+j}\right) .
\end{aligned}
$$

2.3. Determination of the Lagrange Parameters. Now $\ddot{h}_{k m}=0$ implies $h_{k m}=g_{k m}^{1} t+g_{k m}^{0}$ for some constants $\left\{g_{k m}^{1}, g_{k m}^{0}\right\}$, and so, if in addition, we have

$$
h_{k m}\left(\mathbf{p}_{k}(0), \mathbf{p}_{m}(0), 0\right)=\dot{h}_{k m}\left(\mathbf{p}_{k}(0), \mathbf{p}_{m}(0), 0\right)=0,
$$

we could then conclude that $h_{k m}\left(\mathbf{p}_{k}(t), \mathbf{p}_{m}(t), t\right)=0$ for all $t \geq 0$. In other words, if (19) is satisfied, and the Lagrange parameters are chosen such that $\ddot{h}_{k m}=0$, the constraint $h_{k m}=0$ will be satisfied for all $t>0$.

Numerical Robustness: However in order to provide some numerical robustness for the constrained mass-spring model, the Lagrange parameters $\left\{\lambda_{q r}\right\}$ should be selected so as to guarantee that: any small error at time $t_{1}$ (which could arise as the result of a numerical error) in the constraint of the form $h_{k m}\left(\mathbf{p}_{k}\left(t_{1}\right), \mathbf{p}_{m}\left(t_{1}\right), t_{1}\right) \neq 0$ or 
$\dot{h}_{k m}\left(\mathbf{p}_{k}\left(t_{1}\right), \mathbf{p}_{m}\left(t_{1}\right), t_{1}\right) \neq 0$ will decay asymptotically to zero for $t>t_{1}$. Accordingly, we elect to choose the Lagrange parameters such that

$$
\ddot{h}_{k m}+a_{k m} \dot{h}_{k m}+b_{k m} h_{k m}=0
$$

where the numerical robustness parameters $\left\{a_{k m}, b_{k m}\right\}$ are selected so that the roots of the equation

$$
\lambda^{2}+a_{k m} \lambda+b_{k m}=0
$$

are strictly Hurwitz. In addition to guaranteeing asymptotic satisfaction of the constraints, the resulting modes of the constraint dynamics (20) should not be so fast as to cause numerical problems as a result of the differential equations becoming too "stiff".

Now, from (17), we have

$$
\begin{aligned}
\ddot{h}_{k m} & =2\left(\dot{\mathbf{p}}_{k}-\dot{\mathbf{p}}_{m}\right)^{T}\left(\dot{\mathbf{p}}_{k}-\dot{\mathbf{p}}_{m}\right)-\ddot{D}_{k m}+2\left(\mathbf{p}_{k}-\mathbf{p}_{m}\right)^{T}\left(\ddot{\mathbf{p}}_{k}-\ddot{\mathbf{p}}_{m}\right) \\
(22) \quad & =2\left\|\dot{\mathbf{p}}_{k}-\dot{\mathbf{p}}_{m}\right\|^{2}-\ddot{D}_{k m}+2\left(\mathbf{p}_{k}-\mathbf{p}_{m}\right)^{T}\left(\mathbf{f}_{k}-\mathbf{f}_{m}\right)-2\left(\mathbf{p}_{k}-\mathbf{p}_{m}\right)^{T}\left(\hat{\mathbf{f}}_{k}-\hat{\mathbf{f}}_{m}\right)
\end{aligned}
$$

Then from (17), (20) and (22), the Lagrange parameters $\left\{\lambda_{k, m}\right\}$ are given in terms of the numerical robustness parameters $\left\{a_{k m}, b_{k m}\right\}$ from

$$
\left(\mathbf{p}_{k}-\mathbf{p}_{m}\right)^{T}\left(\hat{\mathbf{f}}_{k}-\hat{\mathbf{f}}_{m}\right)=e_{k, m}
$$

where

$$
\begin{aligned}
e_{k, m} & \triangleq\left(\mathbf{p}_{k}-\mathbf{p}_{m}\right)^{T}\left[\mathbf{f}_{k}-\mathbf{f}_{m}+a_{k m}\left(\dot{\mathbf{p}}_{k}-\dot{\mathbf{p}}_{m}\right)+0.5 b_{k m}\left(\mathbf{p}_{k}-\mathbf{p}_{m}\right)\right] \\
& +\left\|\dot{\mathbf{p}}_{k}-\dot{\mathbf{p}}_{m}\right\|^{2}-0.5\left(\ddot{D}_{k m}+a_{k m} \dot{D}_{k m}+b_{k m} D_{k m}\right) .
\end{aligned}
$$

From Theorem 2.2, we have the following result.

Lemma 2.4. For $M \geq 3$, if the two nodes $\left\{\mathbf{p}_{2 i-1}, \mathbf{p}_{2 i}\right\}$ represent a bar in stage $k$ for $2 \leq k \leq M-1$, then

$$
\hat{\mathbf{f}}_{2 i-1}=\lambda_{2 i-1,2 i}\left(\mathbf{p}_{2 i-1}-\mathbf{p}_{2 i}\right) ; \hat{\mathbf{f}}_{2 i}=-\lambda_{2 i-1,2 i}\left(\mathbf{p}_{2 i-1}-\mathbf{p}_{2 i}\right)
$$

where

$$
\lambda_{2 i-1,2 i}=\frac{e_{2 i-1,2 i}}{\left\|\mathbf{p}_{2 i-1}-\mathbf{p}_{2 i}\right\|^{2}}
$$

with $\left\{e_{2 i-1,2 i}\right\}$ given by (24).

At a base (or top) of the $\left(N, S ; P_{1}, P_{2}, \ldots P_{M}\right)$ tensegrity platform, the geometric constraint on any one base (or top) node $\mathbf{p}_{k}$ is coupled to all other base (or top) nodes and to all nodes which define the base (or top) bars. Hence from Theorem 2.2, we have the following result. 
Lemma 2.5. (i) The $4 P_{1}-6$ Lagrange parameters for the base nodes and base bar nodes are given by the vector $\mathbf{v}_{b} \in \mathcal{R}^{4 P_{1}-6}$ which satisfies a linear algebraic equation

$$
\mathbf{F}_{b} \mathbf{v}_{b}=\mathbf{g}_{b}
$$

for some symmetric matrix $\mathbf{F}_{b}$ where

$$
\mathbf{v}_{b}^{T}=\left[\mathbf{v}_{b 1}^{T}, \mathbf{v}_{b 2}^{T}, \mathbf{v}_{b 3}^{T}, \mathbf{v}_{b 4}^{T}\right] ; \mathbf{g}_{b}^{T}=\left[\mathbf{g}_{b 1}^{T}, \mathbf{g}_{b 2}^{T}, \mathbf{g}_{b 3}^{T}, \mathbf{g}_{b 4}^{T}\right]
$$

where vector components are given by

$$
\begin{aligned}
\mathbf{v}_{b 1} & =\left[\lambda_{b_{2 i-1,2 i}} ; i=1,2, . ., P_{1}\right] \in \mathcal{R}^{P_{1}} \\
\mathbf{v}_{b 2} & =\left[\lambda_{b_{1,2 i+1}} ; i=1,2, . ., P_{1}-1\right] \in \mathcal{R}^{P_{1}-1} \\
\mathbf{v}_{b 3} & =\left[\lambda_{b_{3,2 i+3}} ; i=1,2, . ., P_{1}-2\right] \in \mathcal{R}^{P_{1}-2} \\
\mathbf{v}_{b 4} & =\left[\lambda_{b_{2 i+3,2 i+5}} ; i=1,2, . ., P_{1}-3\right] \in \mathcal{R}^{P_{1}-3}
\end{aligned}
$$

and

$$
\begin{aligned}
& \mathbf{g}_{b 1}=\left[e_{b_{2 i-1}, b_{2 i}} ; i=1,2, . ., P_{1}\right] \in \mathcal{R}^{P_{1}} ; \\
& \mathbf{g}_{b 2}=\left[e_{b_{1}, b_{2 i+1}} ; i=1,2, . ., P_{1}-1\right] \in \mathcal{R}^{P_{1}-1} ; \\
& \mathbf{g}_{b 3}=\left[e_{b_{3}, b_{2 i+3}} ; i=1,2, . ., P_{1}-2\right] \in \mathcal{R}^{P_{1}-2} ; \\
& \mathbf{g}_{b 4}=\left[e_{b_{2 i+3}, b_{2 i+5}} ; i=1,2, . ., P_{1}-3\right] \in \mathcal{R}^{P_{1}-3}
\end{aligned}
$$

where $\left\{e_{k, m}\right\}$ are given by (24).

(ii) The $4 P_{M}-6$ Lagrange parameters for the top nodes and top bar nodes are given by the vector $\mathbf{v}_{t} \in \mathcal{R}^{4 P_{M}-6}$ which satisfies a linear algebraic equation

$$
\mathbf{F}_{t} \mathbf{v}_{t}=\mathbf{g}_{t}
$$

for some symmetric matrix $\mathbf{F}_{t}$. The vectors $\left\{\mathbf{v}_{t}, \mathbf{g}_{t}\right\}$ are given by $\left\{\mathbf{v}_{b}, \mathbf{g}_{b}\right\}$ in (28) (30) with $b$ replaced everywhere by $t$.

We shall refer to the matrices $\left\{\mathbf{F}_{b}, \mathbf{F}_{t}\right\}$ as the tensegrity base constraint and the tensegrity top constraint matrices respectively.

Structure of Constraint Matrix: A constraint matrix $\mathbf{F}$ (where $\mathbf{F}=\mathbf{F}_{b}, \mathbf{F}_{t}$ ) in Lemma 2.5 besides being symmetric has an additional sparse characteristic. In particular: if we write

$$
\mathbf{F}=\left[\begin{array}{ll}
\mathbf{F}_{1} & \mathbf{F}_{2}^{T} \\
\mathbf{F}_{2} & \mathbf{F}_{4}
\end{array}\right]
$$

where $\mathbf{F}_{1}$ is of dimension $P_{1} \times P_{1}, \mathbf{F}_{2}$ of dimension $3 P_{1}-6 \times P_{1}$, and $\mathbf{F}_{4}$ is of dimension $3 P_{1}-6 \times 3 P_{1}-6$, then:

(i) The matrix $\mathbf{F}_{1}$ is a diagonal whose diagonal components correspond to the bar length constraints. 
(ii) The matrix $\mathbf{F}_{2}$ is of the form

$$
\mathbf{F}_{2}=\left[\begin{array}{l}
\mathbf{F}_{2,1} \\
\mathbf{F}_{2,2} \\
\mathbf{F}_{2,3}
\end{array}\right] ; \mathbf{F}_{2,1} \in \mathcal{R}^{P_{1}-1 \times P_{1}}, \mathbf{F}_{2,2} \in \mathcal{R}^{P_{1}-2 \times P_{1}}, \mathbf{F}_{2,3} \in \mathcal{R}^{P_{1}-3 \times P_{1}}
$$

Here: (a) the first column of $\mathbf{F}_{2,1}$ is nonzero column whereas the following ( $P_{1}-$ 1) $\times\left(P_{1}-1\right)$ submatrix is diagonal, (b) the first and second columns of $\mathbf{F}_{2,2}$ are zero and nonzero columns respectively while the following $\left(P_{1}-2\right) \times\left(P_{1}-2\right)$ submatrix is diagonal, and (c) the first two columns of $\mathbf{F}_{2,3}$ are zero columns and while in the following $\left(P_{1}-3\right) \times\left(P_{1}-2\right)$ submatrix, the component $[i, j]$ is nonzero for $j=i+2$ or $j=i+3$.

(iii) The matrix $\mathbf{F}_{4}$ generally has some zero terms but is not sparse.

Corollary 2.2. The constraint matrices $\left\{\mathbf{F}_{b}, \mathbf{F}_{t}\right\}$ and the vectors $\left\{\mathbf{v}_{b}, \mathbf{g}_{b}, \mathbf{v}_{t}, \mathbf{g}_{t}\right\}$ of the 2-stage 6-bar tensegrity platform are given by

$$
\mathbf{F}_{b}=\mathbf{F}_{1} ; \mathbf{F}_{t}=\mathbf{F}_{0} ; \mathbf{v}_{b}=\mathbf{v}_{1}, \mathbf{g}_{b}=\mathbf{g}_{1}, \mathbf{v}_{t}=\mathbf{v}_{0}, \mathbf{g}_{t}=\mathbf{g}_{0}
$$

where for $j=0,1$, the $6 \times 6$ matrix $\mathbf{F}_{j}$ and the 6 -vectors $\left\{\mathbf{v}_{j}, \mathbf{g}_{j}\right\}$ are given by

$$
\begin{aligned}
\mathbf{F}_{j}= & {\left[\begin{array}{rrrrrr}
\beta_{1+j, 1+j} & 0 & 0 & \beta_{1+j, 4+j} & \beta_{1+j, 5+j} & 0 \\
0 & \beta_{2+j, 2+j} & 0 & -\beta_{2+j, 4+j} & 0 & \beta_{2+j, 6+j} \\
0 & 0 & \beta_{3+j, 3+j} & 0 & -\beta_{3+j, 5+j} & -\beta_{3+j, 6+j} \\
\beta_{1+j, 4+j} & -\beta_{2+j, 4+j} & 0 & \beta_{4+j, 4+j} & \beta_{4+j, 5+j} & -\beta_{4+j, 6+j} \\
\beta_{1+j, 5+j} & 0 & -\beta_{3+j, 5+j} & \beta_{4+j, 5+j} & \beta_{5+j, 5+j} & \beta_{5+j, 6+j} \\
0 & \beta_{2+j, 6+j} & -\beta_{3+j, 6+j} & -\beta_{4+j, 6+j} & \beta_{5+j, 6+j} & \beta_{6+j, 6+j}
\end{array}\right] } \\
(32) & \mathbf{g}_{j}^{T}=\left[\begin{array}{lllllll}
\lambda_{1+j, 2+j} & \lambda_{1+j, 3+j} & \lambda_{1+j, 5+j} & \lambda_{3+j, 4+j} & \lambda_{3+j, 5+j} & \lambda_{5+j, 6+j}
\end{array}\right]
\end{aligned}
$$

where for $j=0,6$

$$
\begin{aligned}
& \beta_{1+j, 1+j}=2\left\|\mathbf{p}_{1+j}-\mathbf{p}_{2+j}\right\|^{2} ; \beta_{1+j, 4+j}=\left(\mathbf{p}_{1+j}-\mathbf{p}_{2+j}\right)^{T}\left(\mathbf{p}_{1+j}-\mathbf{p}_{3+j}\right) \\
& \beta_{1+j, 5+j}=\left(\mathbf{p}_{1+j}-\mathbf{p}_{2+j}\right)^{T}\left(\mathbf{p}_{1+j}-\mathbf{p}_{5+j}\right) \\
& \beta_{2+j, 2+j}=2\left\|\mathbf{p}_{3+j}-\mathbf{p}_{4+j}\right\|^{2} ; \beta_{2+j, 4+j}=\left(\mathbf{p}_{3+j}-\mathbf{p}_{4+j}\right)^{T}\left(\mathbf{p}_{1+j}-\mathbf{p}_{3+j}\right) ; \\
& \beta_{2+j, 6+j}=\left(\mathbf{p}_{3+j}-\mathbf{p}_{4+j}\right)^{T}\left(\mathbf{p}_{3+j}-\mathbf{p}_{5+j}\right) \\
& \beta_{3+j, 3+j}=2\left\|\mathbf{p}_{5+j}-\mathbf{p}_{6+j}\right\|^{2} ; \beta_{3+j, 5+j}=\left(\mathbf{p}_{5+j}-\mathbf{p}_{6+j}\right)^{T}\left(\mathbf{p}_{1+j}-\mathbf{p}_{5+j}\right) ; \\
& \beta_{3+j, 6+j}=\left(\mathbf{p}_{5+j}-\mathbf{p}_{6+j}\right)^{T}\left(\mathbf{p}_{3+j}-\mathbf{p}_{5+j}\right) \\
& \beta_{4+j, 4+j}=2\left\|\mathbf{p}_{1+j}-\mathbf{p}_{3+j}\right\|^{2} ; \beta_{4+j, 5+j}=\left(\mathbf{p}_{1+j}-\mathbf{p}_{3+j}\right)^{T}\left(\mathbf{p}_{1+j}-\mathbf{p}_{5+j}\right) ; \\
& \beta_{4+j, 6+j}=\left(\mathbf{p}_{1+j}-\mathbf{p}_{3+j}\right)^{T}\left(\mathbf{p}_{3+j}-\mathbf{p}_{5+j}\right) \\
& \beta_{5+j, 5+j}=2\left\|\mathbf{p}_{1+j}-\mathbf{p}_{5+j}\right\|^{2} ; \beta_{5+j, 6+j}=\left(\mathbf{p}_{1+j}-\mathbf{p}_{5+j}\right)^{T}\left(\mathbf{p}_{3+j}-\mathbf{p}_{5+j}\right) \\
& \beta_{6+j, 6+j}=2\left\|\mathbf{p}_{1+j}-\mathbf{p}_{6+j}\right\|^{2}
\end{aligned}
$$


For the 2-stage 6-bar tensegrity platform, a straight forward application of the matrix inversion lemma gives an explicit analytical form for computing the inverse $\mathbf{F}^{-1}$ of a constraint matrix which may provide a time saving in a numerical simulation.

THEOREM 2.3. A constrained particle dynamic model which satisfies the principle of virtual work for a $\left(N, S ; P_{1}, P_{2}, \ldots P_{M}\right)$ tensegrity platform is described by

$$
\ddot{\mathbf{p}}_{k}=\mathbf{f}_{k}+\hat{\mathbf{f}}_{k} ; 1 \leq k \leq 2 N
$$

where the Newtonian forces $\left\{\mathbf{f}_{k}\right\}$ are given in terms of the coordinates $\left\{\mathbf{p}_{k} ; 1 \leq k \leq\right.$ $2 N\}$ by (6), (7).

For $M \geq 3$, the constraint forces on nodes $\left\{\mathbf{p}_{2 i-1}, \mathbf{p}_{2 i}\right\}$ which represent a bar in stage $k$ for $2 \leq k \leq M-1$ are given by

$$
\hat{\mathbf{f}}_{2 i-1}=\lambda_{2 i-1,2 i}\left(\mathbf{p}_{2 i-1}-\mathbf{p}_{2 i}\right) ; \hat{\mathbf{f}}_{2 i}=-\lambda_{2 i-1,2 i}\left(\mathbf{p}_{2 i-1}-\mathbf{p}_{2 i}\right)
$$

where $\lambda_{2 i-1,2 i}$ is given by (26), (24). The constraint forces associated with the base and top are given by

$$
\mathbf{v}_{b}=\mathbf{F}_{b}^{-1} \mathbf{g}_{b} ; \mathbf{v}_{t}=\mathbf{F}_{t}^{-1} \mathbf{g}_{t}
$$

where $\left\{\mathbf{F}_{b}, \mathbf{F}_{t}\right\}$ are the tensegrity constraint matrices with $\left\{\mathbf{v}_{b}, \mathbf{g}_{b}, \mathbf{v}_{t}, \mathbf{g}_{t}\right\}$ given by (28) - (30).

Based on the coordinates of the nodes, the state vector $\mathbf{z}$ of the constrained tensegrity particle dynamic model is given by

$$
\mathbf{z}=\left[\mathbf{p}_{1}, \dot{\mathbf{p}}_{1}, \mathbf{p}_{2}, \dot{\mathbf{p}}_{2}, \ldots, \mathbf{p}_{2 N}, \dot{\mathbf{p}}_{2 N}\right] \in \mathcal{R}^{12 N}
$$

However, as we have shown, in a body frame attached to the base, the positions of the $P_{1}$ base nodes $\left\{\mathbf{p}_{b 1}, \mathbf{p}_{b 2}, \ldots, \mathbf{p}_{b P_{1}}\right\}$ are fixed, and so the dynamic behavior of the tensegrity platform using constrained particle dynamics can be described by a reduced order state vector $\tilde{\mathbf{z}} \in \mathcal{R}^{12 N-6 P_{1}}$. From Lemma 2.3, the dimension of $\tilde{\mathbf{z}}$ is $N+3 P_{M}-6$ greater than the order $2 D$ which would be required if the simulation were expressed in terms of the minimal generalized coordinates. For the 2 -stage 6 -bar tensegrity platform, $N+3 P_{M}-6=9$.

3. Control of a Tensegrity Platform. We now consider the control of a tensegrity platform by adjustments of the lengths $\left\{L_{2 k-1,2 k}\right\}$ of the bars; that is, the control input $\mathbf{u}$ is defined by

$$
\mathbf{u}=\left[u_{1} u_{2}, . ., u_{N}\right]^{T} \in \mathcal{R}^{N} ; u_{k} \triangleq L_{2 k-1,2 k}
$$

and the quality of the control will be measured in terms of how well a performance output $\mathbf{y}$ tracks a desired path $\mathbf{y}_{d}$. For this purpose, we first develop a system of nonlinear differential equations

$$
\dot{\mathbf{z}}=\mathbf{f}(\mathbf{z}, \mathbf{u}) ; \mathbf{y}=\mathbf{h}(\mathbf{z})
$$


which describes the input $\mathbf{u}$, state $\mathbf{z}$ and performance output $\mathbf{y}$ of the platform with respect to a body frame attached to the base.

In general, given the tracking time $T$ and the initial state $\mathbf{z}(0)$, a finite time path tracking problem can be formulated as an approximate "inverse problem" in which a continuous control signal $\mathbf{u}_{d}$ is to be computed from the given trajectory $\mathbf{y}_{d}$ so as to minimize the integral of the tracking error $J$ defined by

$$
J(\mathbf{z}(0), T) \triangleq \int_{0}^{T}\left(\mathbf{y}-\mathbf{y}_{d}\right)^{2} d t .
$$

As a result of the nonlinear nature of the model, no solution to this problem is available, and so instead we formulate a sub-optimal solution. Specifically, we divide the trajectory into $R$ segments with endpoints $\left\{\overline{\mathbf{y}}_{1}, \overline{\mathbf{y}}_{2}, \ldots, \overline{\mathbf{y}}_{R}\right\}$, and seek a sequence of constant inputs $\left\{\overline{\mathbf{u}}_{1}, \overline{\mathbf{u}}_{2}, \ldots, \overline{\mathbf{u}}_{R}\right\}$ such that $\overline{\mathbf{y}}_{i}$ is an equilibrium condition for each given $\overline{\mathbf{u}}_{i}$; that is, such that

$$
\mathbf{0}=\mathbf{f}\left(\overline{\mathbf{z}}_{i}, \overline{\mathbf{u}}_{i}\right) ; \overline{\mathbf{y}}_{i}=\mathbf{h}\left(\overline{\mathbf{z}}_{i}\right)
$$

where $\overline{\mathbf{z}}_{i}$ is the steady state value of the system state. A suboptimal quasi-static solution $\overline{\mathbf{u}}_{d}$ is then defined by the piecewise constant input

$$
\overline{\mathbf{u}}_{d}(t)=\sum_{m=0}^{R-1} \overline{\mathbf{u}}_{m} p\left(\frac{R(t-m)}{T}\right)
$$

where $p(\sigma)=1$ for $0 \leq \sigma<1$, and zero elsewhere. Later, we compare the tracking performance of this quasi-static solution to that achieved using a suboptimal gain scheduling algorithm which incorporates a sequence of "local" feedback controllers defined in terms of the equilibrium values $\left\{\overline{\mathbf{z}}_{i}, \overline{\mathbf{u}}_{i}, \overline{\mathbf{y}}_{i}\right\}$.

3.1. Dynamic Model for Control. As described in [7], control of a tensegrity structure can be achieved by changing the bar lengths $\left\{L_{2 k-2,2 k} ; k=1,2 . ., N\right\}$. A change in the bar length $L_{2 k-2,2 k}$ changes the Lagrange parameter $\lambda_{2 k-2,2 k}$ in (26), (24) which then modifies the bar constraint forces $\left\{\hat{\mathbf{f}}_{2 k-1}, \hat{\mathbf{f}}_{2 k}\right\}$ in (25) to affect a change in the dynamical response (34). As evident in (24), $\lambda_{2 k-2,2 k}$ is a direct function of the square $D_{2 k-1,2 k}$ of the length and its derivatives $\left\{\ddot{D}_{2 k-1,2 k}, \dot{D}_{2 k-1,2 k}\right\}$, and so it is simpler (at least for purposes of analysis) to consider introducing a control signal $u_{k}$ to control $D_{2 k-1,2 k}$. While more general control strategies are possible [7], we restrict consideration in this paper to the following result.

Lemma 3.1. Consider the linear second order controller

$$
\ddot{L}_{2 k-1,2 k}+\alpha_{2 k-1,2 k} \dot{L}_{2 k-1,2 k}+\beta_{2 k-1,2 k} L_{2 k-1,2 k}=\beta_{2 k-1,2 k} w_{k} ; \beta_{2 k-1,2 k} \neq 0 .
$$

Suppose the control signal $w_{k}$ is expressed as a nonlinear function of the length and its derivative $\left\{L_{2 k-1,2 k}, \dot{L}_{2 k-1,2 k}\right\}$ and the independent control input $u_{k}$ according to

$$
w_{k}=L_{2 k-1,2 k}^{-1}\left\{u_{k}-\beta_{2 k-1,2 k}^{-1}\left(\dot{L}_{2 k-1,2 k}\right)^{2}\right\} .
$$


Then $D_{2 k-1,2 k}(t)=L_{2 k-1,2 k}^{2}(t)$ satisfies the linear second order differential equation

$$
\ddot{D}_{2 k-1,2 k}+\alpha_{2 k-1,2 k} \dot{D}_{2 k-1,2 k}+2 \beta_{2 k-1,2 k} D_{2 k-1,2 k}=2 \beta_{2 k-1,2 k} u_{k} .
$$

Furthermore, based on the control strategy (39), (40), the Lagrange parameters $\left\{\lambda_{2 k-1,2 k}\right\}$ are given by

$$
\lambda_{2 k-1,2 k}=\frac{\epsilon_{2 k-1,2 k}}{\left\|\mathbf{p}_{2 k-1}-\mathbf{p}_{2 k}\right\|^{2}}
$$

where

$$
\begin{aligned}
\epsilon_{2 k-1,2 k} \triangleq & \left(\mathbf{p}_{2 k-1}-\mathbf{p}_{2 k}\right)^{T}\left[\mathbf{f}_{2 k-1}-\mathbf{f}_{2 k}+a_{2 k-1,2 k}\left(\dot{\mathbf{p}}_{2 k-1}-\dot{\mathbf{p}}_{2 k}\right)\right. \\
& \left.+0.5 b_{2 k-1,2 k}\left(\mathbf{p}_{2 k-1}-\mathbf{p}_{2 k}\right)\right]+\| \dot{\mathbf{p}}_{2 k-1} \\
& -\dot{\mathbf{p}}_{2 k} \|^{2}-0.5\left(a_{2 k-1,2 k} \dot{D}_{2 k-1,2 k}+b_{2 k-1,2 k} D_{2 k-1,2 k}\right) \\
& -0.5\left(2 \beta_{2 k-2,2 k} u_{2 k-1,2 k}-2 \beta_{2 k-2,2 k} D_{2 k-1,2 k}-\alpha_{2 k-1,2 k} \dot{D}_{2 k-1,2 k}\right) .
\end{aligned}
$$

From Theorem 2.3 and Lemma 2.3, components of the state of the tensegrity platform consist of the $2 D$ positions and velocities of the constrained particles. In addition, from (41), the $N$ bar controllers require the need of a further $2 N$ state components $\left\{D_{2 k-1,2 k}, \dot{D}_{2 k-1,2 k}\right\}$. Therefore in summary, a system of equations in terms of the generalized coordinates and their derivatives with respect to a body frame attached to the base is of the form (36) where $\mathbf{z} \in \mathcal{R}^{N_{D}}$ with

$$
N_{D}=2 D+2 N=2\left(6 N-3 P_{1}+6-3 P_{M}\right) .
$$

However, as we have already mentioned, since the generalized coordinates are not easily obtained, it is preferable to define the state variables $\mathbf{z} \in \mathcal{R}^{N_{S}}$ as the coordinates of particles (after eliminating the base nodes) where

$$
N_{S}=2\left(6 N-3 P_{1}+N\right) \text {. }
$$

For example, for the 2-stage 6-bar platform, $N_{D}=48$ whereas $N_{S}=66$.

3.1.1. 2-Stage 6-Bar Platform. All of the numerical results for control which follow are obtained for a 2-stage 6-bar tensegrity structure in which the performance output $\mathbf{y}$ is defined by

$$
\mathbf{y}(t)=\left[x_{c}(t) y_{c}(t) z_{c}(t) \phi(t) \zeta(t) \omega(t)\right]^{T}
$$

where $\left\{x_{c}, y_{c}, z_{c}\right\}$ are the coordinates of the center of the top, and $\{\phi, \zeta, \omega\}$ are the Euler angles 3-2-1 sequence representing the orientation of the top relative to the base. In this case, $\left\{N=6, M=2 ; P_{1}=P_{2}=3\right\}$ and so the system state $\mathbf{z} \in \mathcal{R}^{N_{S}}$ where $N_{S}=66$. (If the generalized coordinates could be found, then a state space representation of $N_{D}=48$ states could be used.) Because of the complexity of the system and space limitations, we only include a summary of the numerical results. A full description of the simulation results data is available [19]. 
3.2. Neural Network Approximation. The nonlinear constrained massspring system (36) is a passive system subject to geometric constraints. We therefore have the following result.

Lemma 3.2. Suppose the Lagrange parameters $\left\{\lambda_{2 k-1,2 k}\right\}$ of the constrained mass-spring model are given by (42), (43). Then given any initial state $\mathbf{z}(0)$ and any constant control signal $\overline{\mathbf{u}}=\left[\bar{u}_{1}, \bar{u}_{2}, \ldots, \bar{u}_{N}\right]^{T}$ with $\bar{u}_{k}>0$ for all $k$, the steady state value $\overline{\mathbf{z}}$ of the platform state is bounded, and the steady state bar lengths $\left\{\bar{L}_{k}\right\}$ are given by

$$
\bar{L}_{k}=\sqrt{\bar{u}_{k}} ; k=1,2, . ., N
$$

Note that in steady state (ie zero velocity), half of the steady state components of $\overline{\mathbf{z}}$ are zero. For example, for the 2-stage 6-bar platform, (37) define a nonlinear algebraic relationship between 6 constant input values (components of $\left\{\overline{\mathbf{u}}_{i}\right\}$ ), 33 constant internal values (non-zero components of $\left\{\overline{\mathbf{z}}_{i}\right\}$ ) and 6 constant output values (components of $\left\{\overline{\mathbf{y}}_{i}\right\}$ ).

Suppose from (37), there exists an invertible function $\mathcal{H}$ such that $\overline{\mathbf{y}}_{i}=\mathcal{H}\left(\overline{\mathbf{u}}_{i}\right)$. Then given points $\left\{\overline{\mathbf{y}}_{1}, \overline{\mathbf{y}}_{2}, \ldots, \overline{\mathbf{y}}_{R}\right\}$ on the desired path, a suboptimal tracking solution (38) is given from the inverse function

$$
\overline{\mathbf{u}}_{i}=\mathcal{H}^{-1}\left(\overline{\mathbf{y}}_{i}\right) .
$$

The existence of an inverse is based on the assumption that for any given constant input $\overline{\mathbf{u}}_{i}$ (corresponding to a given set of $N$ bar lengths), there exists a unique steady state output $\overline{\mathbf{y}}_{i}$. Even though asymptotic stability is guaranteed, sufficient conditions for uniqueness are still not available.

However, even if an inverse were known to exist, an explicit form would be unlikely. Therefore based on the assumption that an inverse exists, we seek a artificial neural network approximation [14] to $\mathcal{H}^{-1}$. As is well-known, a neural network must be "trained" using a data obtained from input-output data of the steady state system (37). In [15], it is suggested that a multi-layered feed-forward neural network with a back-propagation learning rule provides the best results. In each specific application, the problem however is then to determine the number of layers and neurons noting that both accuracy and convergence suffer if the numbers of neurons and layers are either too small or too large.

3.2.1. Training Data for 2-Stage 6-Bar Platform. The training data consisted of 1456 pairs of steady state input-output data $\left\{\overline{\mathbf{y}}_{i}, \overline{\mathbf{u}}_{i}\right\}$ obtained from the simulation for various bar lengths $\left\{L_{1,2}, \ldots, L_{11,12}\right\}$ that corresponded to the $\{0 \%, \pm 10 \%$, $\pm 20 \%\}$ of the nominal lengths $L_{i, j}=2$. The input-output data however was first normalized so that inputs and outputs had zero mean and unit variance to assure that the input data evenly contributed to the output data. 
The hyperbolic tangent sigmoid function was chosen, and the neuron network was trained by using Matlab and Neural Network Toolbox ${ }^{1}$. Training was regarded as having successfully completed once the mean square error between the output from the network and the training set was less than $5 E-06$. The "optimal" neuron network consisted of 3 layers (2 hidden and 1 output). Each hidden layer had 30 neurons and the output layer had 6 neurons.

In order to test the quality of the neural network approximation, new data was generated based on bar lengths adjusted at $\pm 15 \%$ (which is within the range of the training data) and $\pm 25 \%$ (which is outside the range of the training data) of their nominal lengths. Each set of the testing data had 729 pairs of input-output testing data. The error between the true output and that generated by the neural network had mean square errors equal to $2.34 E-05$ and $1.40 E-03$ for the testing at $\pm 15 \%$ and $\pm 25 \%$ respectively. Inspite of the relatively large error for the $\pm 25 \%$ data set, the neural network approximation proved to be reasonably robust and indicates a "smoothness" property of the nonlinear input-output mapping $\mathcal{H}$. We also investigated the numerical sensitivity of the neural network to small changes in control signal as a result of the neural network approximation, and found that the nonlinear model was relatively insensitive to such changes especially with respect to changes in the Euler angle variables. Once again this result is indicative of a "smoothness" property of the nonlinear input-output mapping $\mathcal{H}$.

3.2.2. Tracking Performance of 2-Stage 6-Bar Platform. In order to evaluate the tracking performance, we consider a linear path $\mathbf{y}_{d}(t)$ defined by

$$
\mathbf{y}(t)=\mathbf{y}_{R}-\frac{\left(\mathbf{y}_{R}-\mathbf{y}_{1}\right)(T-t)}{T}
$$

where

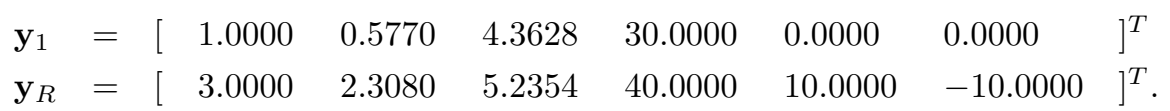

Various simulations were then performed with the path divided into $R=10,20$ and 40 segments with time increments $\triangle t=0.625,1.25,2.5$, and 5 seconds corresponding to a tracking time $T=R \triangle t$ seconds. The mean square tracking errors are given in Table 1.

We make the following observations: (i) For a constant time increment $\Delta t$, tracking performance improves as $R$ increases, (ii) for a constant number of increments $R$, tracking performance improves as $\Delta t$ increases, and (iii) for a constant number of tracking time $T$, tracking performance improves as $R$ increases ( $\triangle t$ decreases). Two particular results $(R=10, \triangle t=5)$ and $(R=40, \triangle t=2.5)$ labelled 1 and 2 respectively are shown in Fig 3 and 4 . The one segment or step response characteristics

\footnotetext{
${ }^{1}$ Matlab and Neural Network toolbox is the registered trademark of of The Math works, Inc., http://www.mathworks.com
} 
TABLE 1

Tracking error with various numbers of segments $R$ and time increments $\Delta t$

\begin{tabular}{c|c|c|c|c}
\hline \multirow{2}{*}{ No. of segments } & \multicolumn{4}{|c}{ Mean Square Error } \\
\cline { 2 - 5 } & $\triangle t=0.625$ & $\triangle t=1.25$ & $\triangle t=2.5$ & $\triangle t=5$ \\
\hline \hline 40 & $1.59 \mathrm{E}-02$ & $1.29 \mathrm{E}-02$ & $1.00 \mathrm{E}-02$ & $1.06 \mathrm{E}-2$ \\
20 & $2.18 \mathrm{E}-02$ & $1.58 \mathrm{E}-02$ & $1.39 \mathrm{E}-02$ & $1.67 \mathrm{E}-2$ \\
10 & $2.58 \mathrm{E}-02$ & $2.17 \mathrm{E}-02$ & $1.85 \mathrm{E}-02$ & $2.58 \mathrm{E}-02$ \\
\hline
\end{tabular}

corresponding to $R=1$ as illustrated in Fig 5 and Fig 6 are highly oscillatory with an approximate "settling time" of 50 seconds. Note also that the tracking performance significantly deteriorated for $T<50$ seconds, and the inability to further reduce the tracking time is consistent with the open loop nature of the quasi-static approach to tracking.

3.2.3. Gain Scheduling Algorithm. We now show how a "gain scheduling" control algorithm can lead to a reduction in tracking time compared with the quasistatic approach without a deterioration in tracking mean square error. Specifically, we consider a finite number $R$ of constant operating points as parameterized by the scheduling variable $\left\{\alpha_{i} ; i=1,2, . ., R\right\}$ such that

$$
\mathbf{0}=\mathbf{f}\left(\mathbf{z}\left(\alpha_{i}\right), \mathbf{u}\left(\alpha_{i}\right), \alpha_{i}\right) ; \mathbf{y}\left(\alpha_{i}\right)=\mathbf{h}\left(\mathbf{z}\left(\alpha_{i}\right), \mathbf{u}\left(\alpha_{i}\right), \alpha_{i}\right)
$$

and seek a nonlinear controller of the form

$$
\mathbf{u}(t)=\mathbf{K}(\alpha) \mathbf{z}(t)+\{\mathbf{u}(\alpha)-\mathbf{K}(\alpha) \mathbf{z}(\alpha)\}
$$

The gains $\{\mathbf{K}(\alpha)\}$ at $\alpha=\alpha_{i}$ were determined from the linearized system at $\mathbf{z}=\mathbf{z}\left(\alpha_{i}\right)$ based on a LQR solution, and $\{\mathbf{K}(\alpha)\}$ for $\alpha_{i}<\alpha<\alpha_{i+1}$ were determined using the interpolated method in [10].

"Local" gains $\{\mathbf{K}(\alpha)\}$ were determined at $R=3$ operating points for 10 and 20 segments, and at $R=5$ operating points for 40 segments. The $\mathbf{z}\left(\alpha_{i}\right)$ were determined by simulation with $\mathbf{u}=\mathbf{u}_{i}$. In each case, a "local" LQR design resulted in a "local" closed loop responses having a "settling time" of approximately 3 seconds. The corresponding mean square tracking error of path tracking for different segment numbers and time increments $\Delta t$ are presented in Table 2 .

The simulations in Fig 7 and Fig 8 illustrate the tracking responses when the $(R=40, \triangle t=1.25)$ for a tracking time of $T=50$ seconds. Linear gains $\left\{\mathbf{K}\left(\alpha_{i}\right)\right\}$ were determined at path nodes 2,10,20,30 and 41 and the other gains were obtained by interpolation. The simulations in Fig 9 and 10 are also for $(R=40, \triangle t=1.25)$ and a tracking time of $T=50$ seconds. However in this case, only one linear gain was calculated (at path node 2) and this gain was then used at all other nodes. While some 
TABLE 2

Mean square tracking errors for gain scheduling algorithm

\begin{tabular}{c|ccc}
\hline No. of segments & \multicolumn{4}{|c}{ Mean Square Error } \\
& $\triangle \mathrm{t}=0.625$ & $\triangle \mathrm{t}=1.25$ & $\triangle \mathrm{t}=2.5$ \\
\hline \hline 40 & $3.8970 \mathrm{E}-03$ & $1.1774 \mathrm{E}-03$ & $1.7942 \mathrm{E}-03$ \\
20 & $1.5383 \mathrm{E}-03$ & $3.7862 \mathrm{E}-03$ & $5.4167 \mathrm{E}-03$ \\
10 & $3.2965 \mathrm{E}-02$ & $1.0788 \mathrm{E}-02$ & $1.8888 \mathrm{E}-02$ \\
\hline
\end{tabular}

deterioration is now evident compared with the interpolated approach, the response is significantly better than the quasi-static results in Fig 3 and Fig 4. "The mean square tracking errors of path tracking corresponding to various numbers of segments and time increments $\delta t$ are presented in Table 2.

From Table 2, it is evident that the nonlinear gain scheduling algorithm improves the tracking performance compared to the quasi-static approach since both the mean square tracking error and the tracking time are reduced. For example, when $R=10$ and $\delta t=1.25$, the mean square error of $1.08 E-02$ is obtained for a tracking time $T$ of approximately 15 seconds. However, without gain scheduling ( see Table 1 ), the tracking time $T$ must be greater than 100 seconds when $R=40$ and $\delta t=1.25$ in order to achieve a mean square tracking error of $1.00 E-02$.

3.3. A Tensegrity Flight Simulator. A recent paper [18] has proposed the use of a 2-stage 6-bar tensegrity platform for a flight simulator as an alternative to a Stewart Platform. As both approaches provide important contributions to design, we now point out some differences and similarities. In particular:

(i) In [18], the model is based on a symmetrical mode of operation. This enables the geometry of the structure to be expressed in terms of only two angles $\delta, \alpha$ which reduces the degrees of freedom to six. The six independent degrees of freedom are then selected as the variables $\mathbf{y}^{T}=\left[x_{c}(t), y_{c}(t), z_{c}(t), \phi(t), \zeta(t), \omega(t)\right]$ in (44). In this paper, the model does not assume a symmetrical mode of operation, and as a result has a total of $D=18$ degrees-of-freedom.

(ii) In [18], the resulting dynamical equations of state dimension 12 are expressed in the form

$$
\mathbf{M}(\mathbf{y}) \ddot{\mathbf{y}}+\mathbf{c}(\mathbf{y}, \dot{\mathbf{y}})+\mathbf{A}(\mathbf{y}) \mathbf{T}(\mathbf{y})+\hat{\mathbf{G}}=\mathbf{0}
$$

using Lagrange methodology where $\mathbf{M}(\mathbf{y})$ is the system inertia matrix. No dynamics are included for control action. In this paper, a constrained particle dynamic model (ignoring control dynamics) of order 54 is developed. (Recall that if 18 independent generalised coordinates could be found, then in principle, a model of order 36 is possible.) A particle dynamic model can only provide an approximation of the dynamical behaviour of full kinematic model where the degree of approximation depends on the 
rotational inertial effects of the bars. Some numerical simulations in ([19]) demonstrate these differences are not large for 'normal' modes of operation of the platform.

(iii) In [18], the control variables are the rest lengths $\left\{\ell_{m k}^{0}\right\}$ of the six 'saddle strings' $\{[6,10],[10,4],[4,12],[12,2],[2,8],[8,6]\}$ in Fig 2 whereas in this paper, the control variables are the lengths of the six bars. For an application as a flight simulator, it is justifiable argued in [18] that string (or tendon) control eliminates the need for telescopic bars (or struts). The resulting dynamic response is also likely to be quicker. Consequently, in this paper, open loop dynamics for the bar controller have been included in the model. In terms of the model equations (8), adjustments to the rest lengths of cables directly effects (some of) the string forces $\left\{\mathbf{f}_{k}\right\}$ which then indirectly affects the constraint forces $\left\{\hat{\mathbf{f}}_{k}\right\}$ whereas adjustments to the bar lengths directly effects the constraint forces $\left\{\hat{\mathbf{f}}_{k}\right\}$ which then indirectly affects the string forces $\left\{\mathbf{f}_{k}\right\}$. For applications that require a larger 'work space', telescopic bars would be required, and so a control strategy which incorporates both tendon and strut control is worthy of investigation. Preliminary work which looked at localized linear particle dynamic models have indicated that control using bar lengths was 'more controllable' (in terms of the size of the minimum singular value of the controllability grammian) than control using rest lengths of the 'saddle strings'.

(iv) The equilibrium configuration of the platform structure as determined by both the particle dynamic model and the full kinematic model are equivalent. However in [18], equilibrium configurations are only possible for a symmetric structure whose geometry is described by two angles. The control methodology in this paper is based on developing a neural network approximation of the inverse of the function $\mathcal{H}$ which describes the equilibrium configuration of the platform in terms of steady state values of control (the six bar lengths), states (the coordinates of bars) and outputs (the six variables in $\mathbf{y}$ ). Since both models give the same equilibrium configuration, these results are valid for either model (though transient response characteristics differ). A similar neural network approximation can be also be undertaken when the inputs are the rest lengths of the 'saddle strings'. This work is still under investigation.

4. Conclusion. This paper has developed a passive nonlinear constrained particle dynamic model of a tensegrity platform as the basis for the development of a path tracking algorithm using an artificial neural network. The explicit form of Lagrange multipliers which result from the constraints are derived, and can be used to provide a model for the internal forces exerted in the bars. The platform has potential use as a flight simulator or a smart structure, but many other applications exist. A comparison with recent work on control of a tensegrity flight simulator has been given. The disadvantage of requiring a higher state dimension when compared with the number of independent degrees of freedom is balanced by the fact that the equations are simple to derive and simulate. 
The artificial neural network provides a solution to the inverse problem of determining the constant control that moves the structure to a desired steady state output. Even though the neural network only approximates the inverse input-output function, it is shown to be reliably robust in its performance using both quasi-static and gain scheduling control algorithms. In particular, it was found that inclusion of a constant linear feedback control law greatly improved the performance of the quasi-static algorithm.

One open problem that remains is to be able to design an appropriate path between two given platform equilibrium conditions so that during path tracking, given constraints on string tension and bar lengths are not exceeded. A more fundamental problem is to be able to establish conditions under which the steady state nonlinear input-output function $\mathcal{H}$ is invertible. Some recent results [20] may provide further insights into this problem. A related problem is to be able to analyse 'smoothness' properties of $\mathcal{H}$ in order to provide a better understand as to why the neural network provided such an accurate approximation of $\mathcal{H}^{-1}$.

\section{REFERENCES}

[1] D. E. IngBer, Architecture of Life, Scientific American, Jan., pp. 48-57, 1998.

[2] R. Connelly and A. Black, Mathematics and Tensegrity, American Scientist, Vol. 86, MarApr, 1998.

[3] H. Furuya, Concept of Deployable Tensegrity Structures in Space Applications, Int. J. Space Structures, 7:2(1992), pp. 143-151.

[4] R. Adhikari, R. E. Skelton, and W. J. Helton, Mechanics of Tensegrity Beams, UCSD, Structural Systems \& Contr. Lab., Rep. No. 1998-1, 1998.

[5] D. Williamson and R. E. Skelton, A General Class of Tensegrity Systems: Geometric Definition, Engineering Mechanics for the 21st Century, ASCE Conference, La Jolla, California, May, 1998.

[6] D. Williamson and R. E. Skelton, A General Class of Tensegrity Structures: Topology and Prestress Equilibrium Analysis, AIAA J. Guidance, Contr., \& Dynamics (to be published).

[7] N. Kanchanasaratool and D. Williamson, Modelling and Control of Class NSP Tensegrity Structures, Int. J. Control, 75:2(2002), pp. 123-139.

[8] N. Kanchanasaratool and D. Williamson, Modelling of Class NSP Tensegrity Structures, 6th Int. Conf. on Control, Auto., Vision \& Robotics, Singapore, 2000.

[9] N. Kanchanasaratool and D. Williamson, Control of Class NSP Tensegrity Structures, 6th Int. Conf. on Control, Auto., Vision \& Robotics, Singapore, 2000.

[10] D. J. Stilwell and W. J. Rugh, Interpolation of Observer State Feedback Controllers for Gain Scheduling, IEEE Trans. Auto. Cntr., 44:6(1999), pp. 1125-1229.

[11] G. Cybenko, Approximation by Superpositions of a Sigmoidal Function, Math. Contr., Signals, Syst., 2(1989), pp. 303-314.

[12] H. Goldstein, Classical Mechanics, Addison-Wesley, Reading, MA, 1950.

[13] J. Garcia de Jalon and E. BAyo, Kinematics and Dynamic Simulation of Multibody Systems, Springer-Verlag, New York, 1998.

[14] K. M. Hornik, M. Stinchcombe, And H. White, Multilayer Feedforward Networks as Universal Approximations, Neural Networks, PWS Publishing, Boston, 1996.

[15] G. Krishnaswamy, M.H, JR, G. B. Anderson, Structured Neural Network Appraoch to Robot 
Motion Control, Proc. IEEE Int. Joint Con. Neural Networks, Singpore, 1991.

[16] M. T. Hagan, H. B. Demuth, and Mark Beale, Neural Network Design, PWS Publishing, Boston, 1996.

[17] S. Haykin, Neural Networks: A Comprehensive Foundation, Prentice Hall, New Jersy, 1999.

[18] C. Sultan, M. Corless, and R.E. Skelton, Tensegrity Flight Simulator, J. Guidance, Control, and Dynamics, 23:3(2000), pp. 1055-1064.

[19] N. Kanchanasaratool, A Simulation Study of a 2-Stage 6-Bar Tensegrity Platform, http://hilbert.anu.edu.au/ narong/platform.html.

[20] D. Williamson, R. E. Skelton, and J. H. Han, Equilibrium Conditions of a Tensegrity Structure, 3rd World Conf. o n Structural Control, Como, Italy, April 2002. 


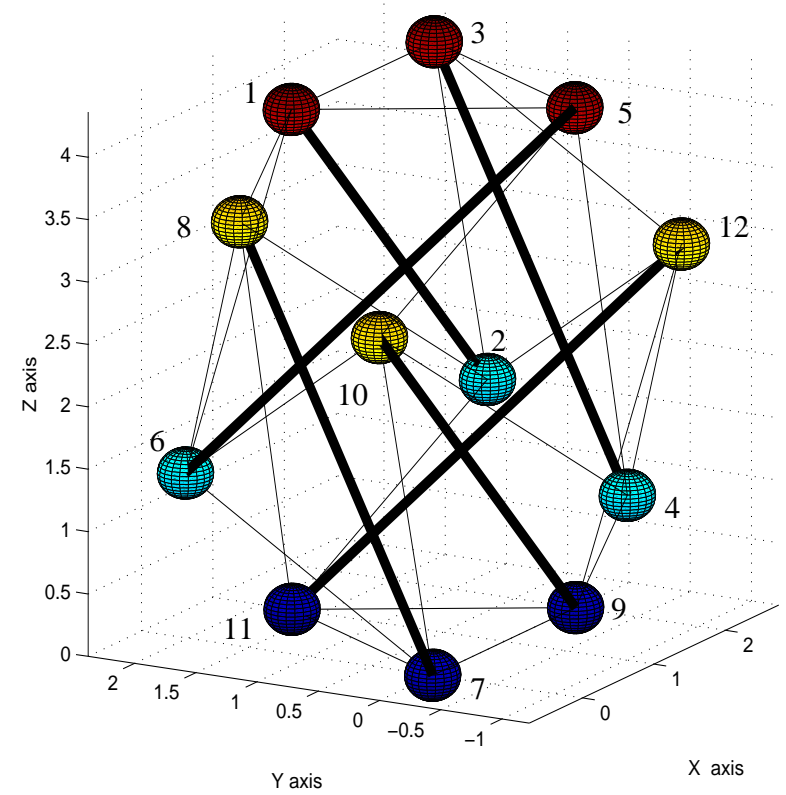

Fig. 1. A 2-stage 6-bar $(6,24 ; 3,3)$ class 1 tensegrity structure.

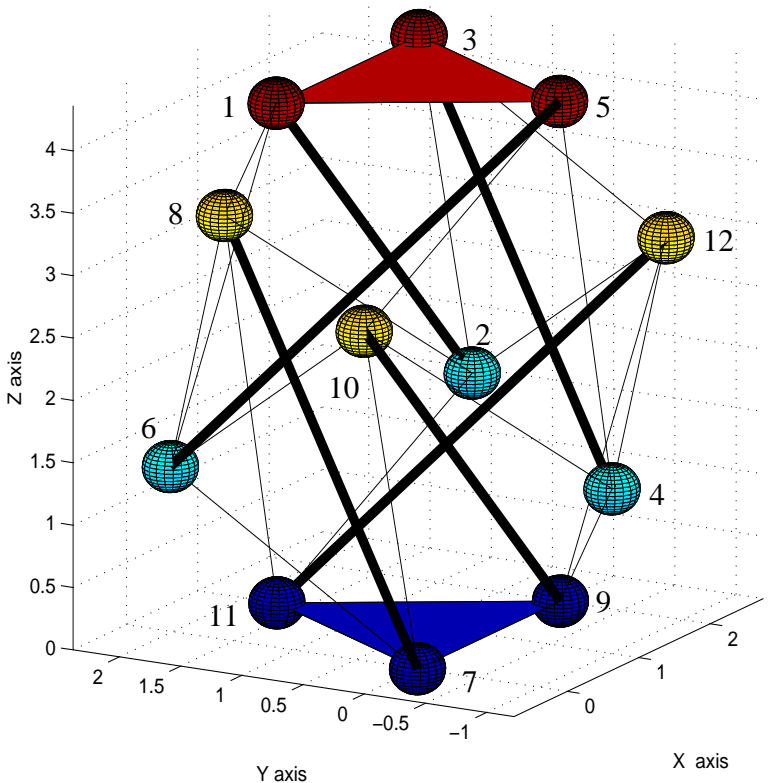

FIG. 2. A 2-stage 6-bar class 2 tensegrity platform. 


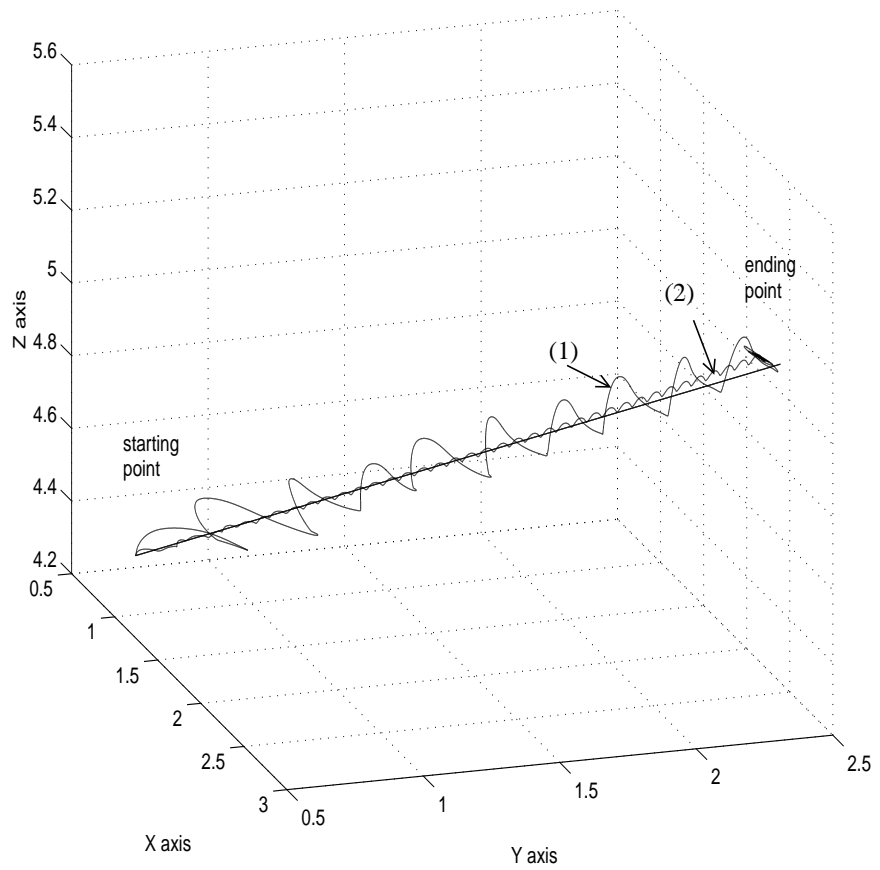

FIG. 3. Path tracking characteristics for: (1) $(R=10, \triangle t=1.25)$ and (2) $(R=40, \Delta t=1.25)$.
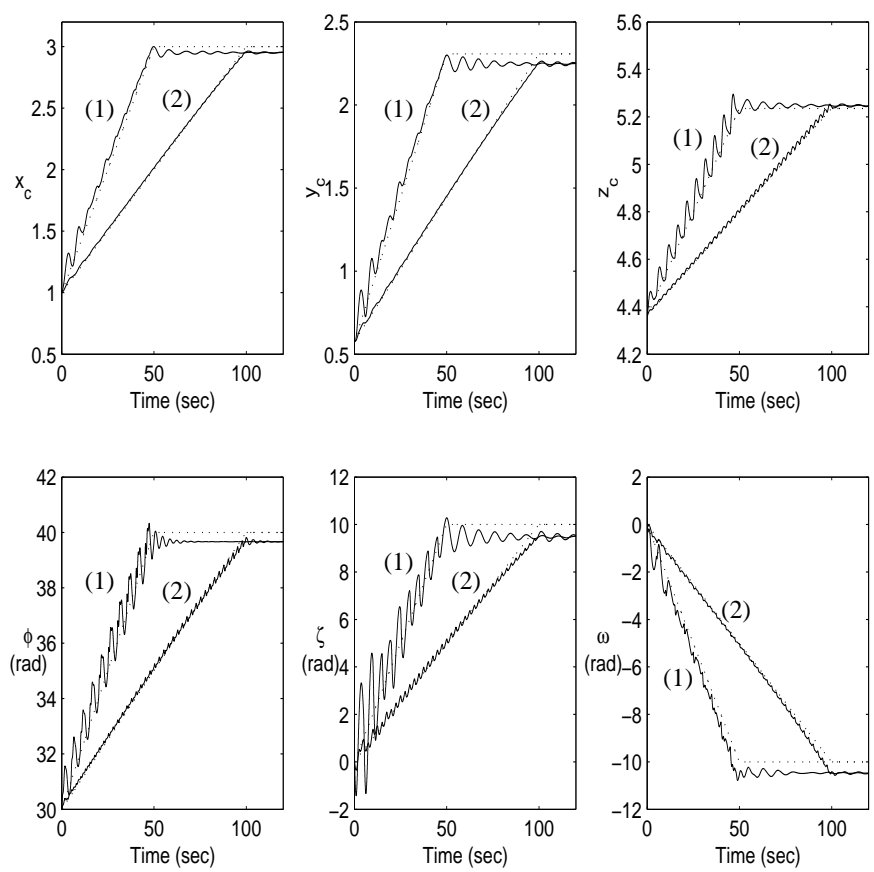

FIG. 4. Path tracking characteristics for: (1) $(R=10, \triangle t=1.25)$ and (2) $(R=40, \triangle t=1.25)$. 


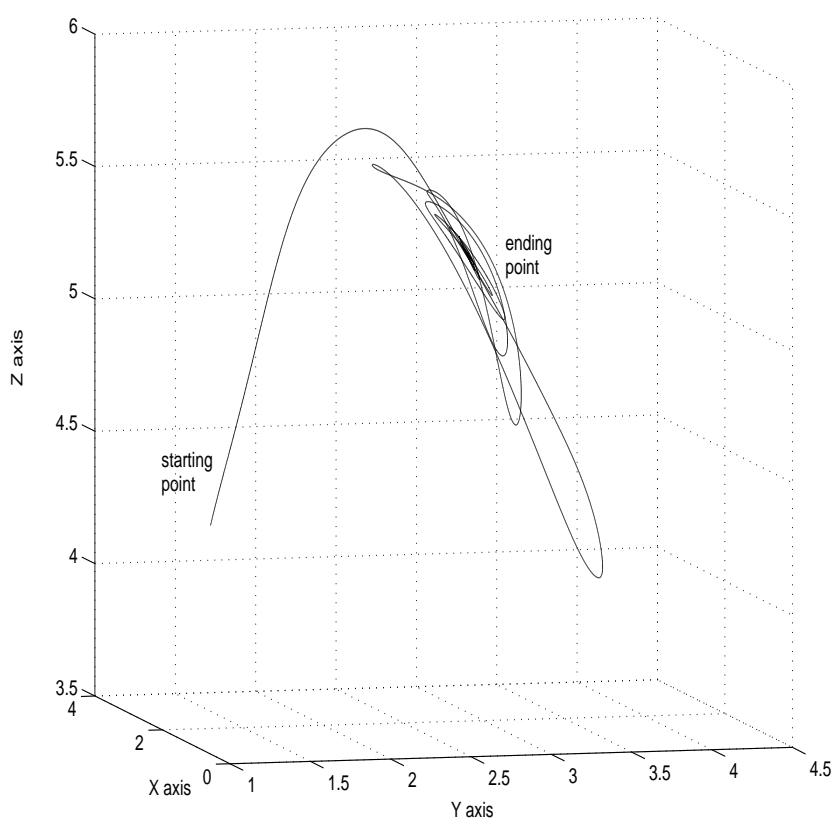

FIG. 5. Tracking response when $R=1$.
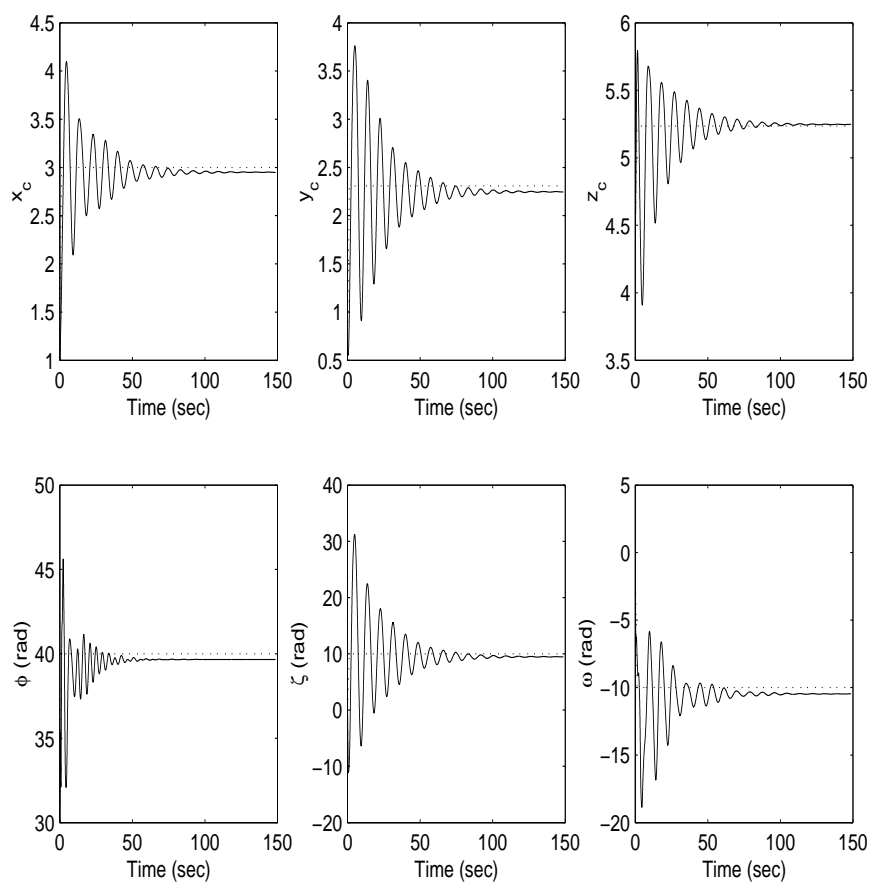

FIG. 6. Tracking response when $R=1$. 


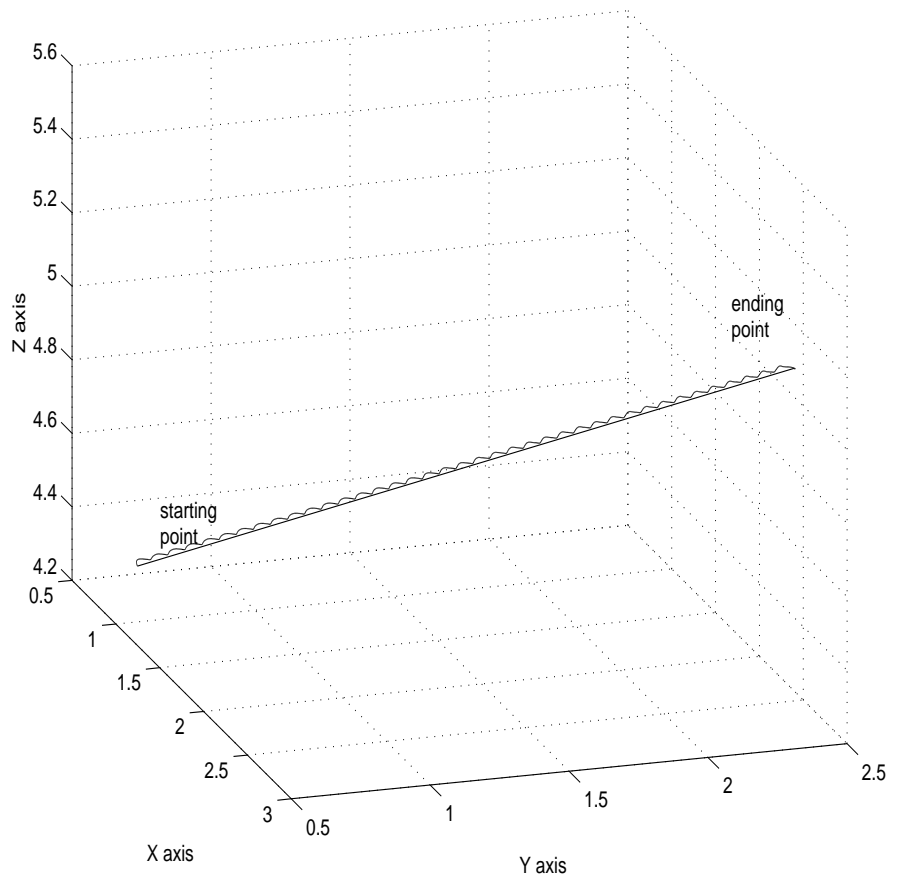

FIG. 7. Path tracking response of gain scheduled system where $R=40, \Delta t=1.25$.
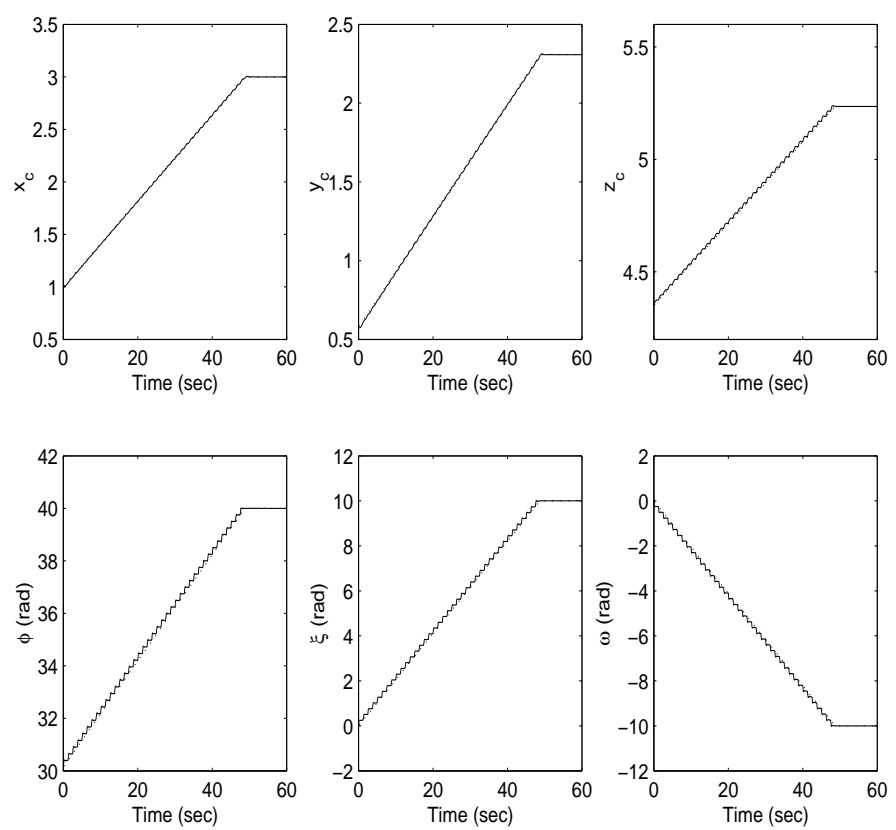

FIG. 8. Path tracking response of gain scheduled system where $R=40, \triangle t=1.25$. 


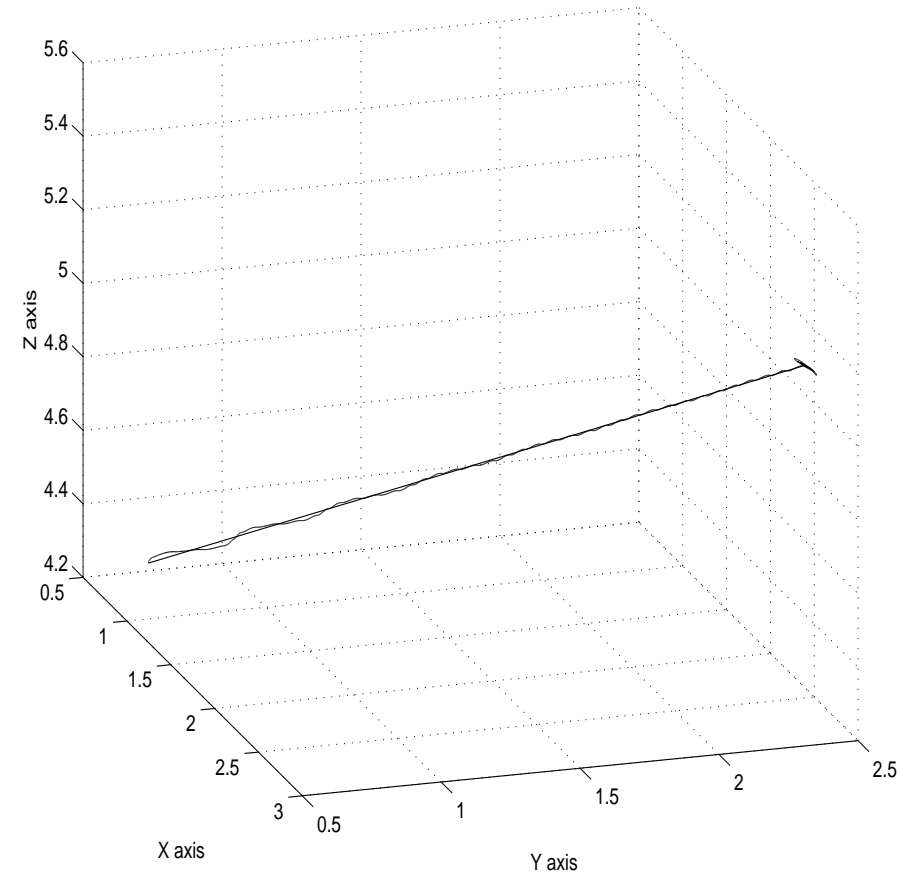

FIG. 9. Path tracking responses in Cartesian coordinate space for fixed gain.
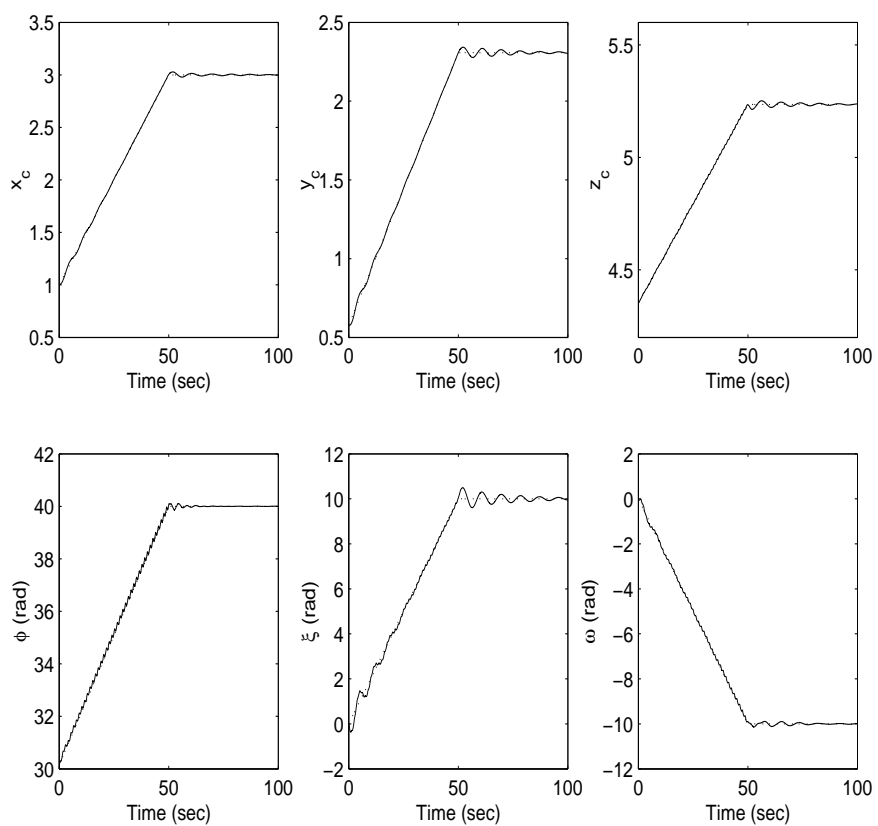

FIG. 10. Path tracking responses in time domain for fixed gain. 